\title{
Click frequency as a stimulus intensity parameter*
}

\author{
DONALD MELTZER, MARK A. MASAKI \\ and \\ BRUCE R. NIEBUHR \\ Southern Illinois University, Carbondale, Ill. 62901
}

Rats were conditioned to respond on the same schedule of reinforcement in the presence of two click stimuli. They consistently responded at higher rates during a 9 clicks/sec stimulus than during a 3 clicks/sec stimulus. However, when the difference between stimuli was increased to 18 clicks/sec vs 3 clicks/sec, the difference between response rates in the two stimuli did not increase. Click frequency was interpreted as a stimulus intensity parameter, but theoretical accounts of stimulus intensity effects do not seem to account for these results.

Although clicks are frequently used as an auditory stimulus in studies of animal discrimination learning, there has been relatively little effort devoted to the study of how parametric variables of the click stimulus affect Ss. Mostofsky, Shurtleff, \& Margolius (1964) determined the generalization gradient for click frequency in both rats and humans and found that they were quite similar. They also calculated difference thresholds for click frequency in the two species based on their generalization gradients and found that the threshold was relatively constant at frequencies between 20 clicks/sec and 80 clicks/sec and was equal to approximately 0.12 . Their generalization gradients were symmetrical, and peak response probability occurred when the training stimulus was presented. In a more recent experiment, Saunders (1969) studied the cat's ability to discriminate click intensity. He conditioned cats to make an avoidance response to one level of click intensity and to withhold a response in the presence of another click intensity. A steady click intensity served as the background stimulus, and Saunders was able to establish the difference threshold for click intensities.

It was not clear from these studies whether there were any parameters of the click stimulus which would differentially affect the performance of the same response, as might be the case when a stimulus intensity effect was present. There are several ways in which investigators have demonstrated stimulus intensity effects in instrumental conditioning in the past, but we used a method that had been employed previously by Gray (1965). Gray conditioned rats to barpress for food reinforcement in a free operant situation. Five different intensities of white noise were used as discriminative stimuli, with the same variable-interval schedule in effect during each of the stimuli. Silence was the stimulus during the extinction interval. Each rat was exposed to silence as well as to all five noise intensities, and Gray

*This article is sponsored by James H. McHose, who takes full editorial responsibility for it. found that response rate increased as the intensity of the discriminative stimulus was increased.

This study examined the effect of click frequency in an experimental procedure which would reveal any stimulus intensity effects the stimulus might have.

\section{PROCEDURE}

Six male hooded rats, approximately 90 days old at the beginning of the experiment, were used as Ss. They had free access to food at all times except while in the experimental chamber. Water was used as the reinforcement during the experiment, and each $S$ had free access to water for $15 \mathrm{~min}$ following each experimental session. One $S$ failed to learn the discrimination, and its data are not included in this report.

The experimental chamber was a commercially produced unit (Lehigh Valley 1417) with internal dimensions of $7.5 \times 12 \times 8 \mathrm{in}$. The lever had an operating pressure of $20 \mathrm{~g}$ and was $1.5 \mathrm{in}$. above the floor and $2.0 \mathrm{in}$. from the left side of the chamber. Water reinforcement was delivered by a 0.06 -cc dipper at the base of the front wall and midway between the sides of the chamber. A 2.5-in. speaker was mounted on the outside of the left wall of the chamber and was centered $2.0 \mathrm{in}$. from the front wall and 4.0 in. above the grid floor. Clicks were generated by a modified Scientific Prototype click-flash generator (Model 4041-J), and frequency was calibrated on a Tektronix $561 \mathrm{~A}$ oscilloscope. The click output included one peak with a duration of $6.0 \mathrm{msec}$ and a number of secondary peaks which lasted for an additional $10.0 \mathrm{msec}$. Noise intensity was measured with a General Radio Company 1551-c sound-level meter. White noise was always present in the experimental room, and the sound level within the experimental chamber when the white noise was on and the blower used to ventilate the chamber was operating was approximately $84 \frac{1}{2} \mathrm{~dB}$. Although the sound-level meter could not measure the peak transient intensity of the click accurately, the overall sound intensity rose to $861 / 2 \mathrm{~dB}$ when the 3 clicks/sec stimulus was presented and reached $92 \mathrm{~dB}$ when the 9 clicks/sec stimulus was presented.

After 3 days of continuous reinforcement during a 3 clicks $/ \mathrm{sec}$ stimulus, the Ss were reinforced on a variable-interval (VI) 45-sec schedule during both of the click stimuli. Each stimulus presentation lasted for $3 \mathrm{~min}$ and was followed by a 15-sec period during which no clicks were presented and no responses were reinforced. Only after $15 \mathrm{sec}$ had passed without the $\mathrm{S}$ making a response was the next click stimulus presented. There were a total of 14 click stimulus intervals during the session, 7 of which were intervals during which the stimulus was 3 clicks/sec and the other 7 of which were intervals during which the stimulus was 9 clicks/sec. The schedule of reinforcement was changed to VI 1 min during the third session and remained at that value for the rest of the experiment. The discriminative stimuli remained at 3 clicks/sec and 9 clicks/sec for 52 sessions in Stage 1, after which they were changed to 3 and 18 clicks/sec for 15 sessions in Stage 2. The stimuli were returned to 3 and 9 clicks/sec for the last 15 sessions of the experiment in Stage 3.

\section{RESULTS}

Mean response rates in the presence of each stimulus were calculated for the last 15 sessions of Stage 1, the 15 sessions of Stage 2, and the 15 sessions of Stage 3. These data were then analyzed in a two-factor analysis of variance to see if response rate differed in the presence of the $\mathrm{S}-$ and in each of the click frequencies and to see if response rates differed in different stages of the 

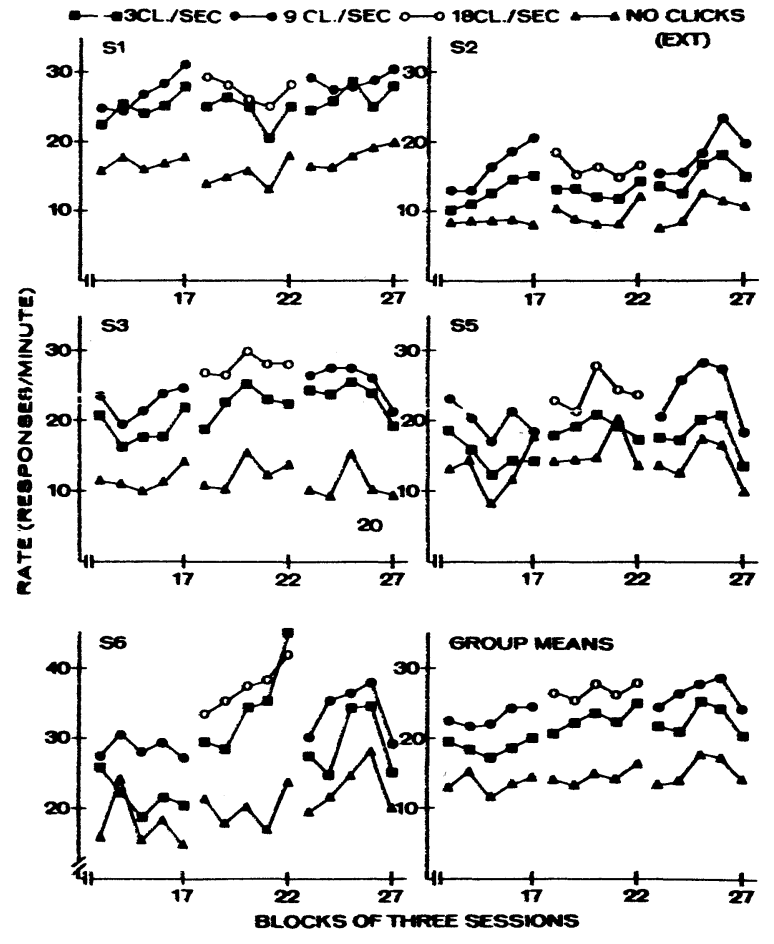

Fig. 1. The response rates of each $S$ during the presentation of each stimulus are shown above. An extinction schedule was always in effect during the no-click condition, and a VI 1-min schedule of reinforcement was always in effect during each of the click stimuli.

experiment. Stimulus condition did have a significant effect on response rate $(F=161.57, \mathrm{df}=2 / 8, \mathrm{p}<.01)$ and, as Fig. 1 shows, Ss consistently responded at lower rates during the $\mathrm{S}-$ than during either of the two $\mathrm{S}+\mathrm{s}$. Figure 1 also shows that the Ss responded at a higher rate during the higher click frequency $S+$ in all stages of the experiment. Stage of the experiment did not have a significant effect on response rate, and the interaction between stimuli and stage of the experiment also failed to reach significance.

An analysis of the mean difference in response rate during the two $S+s$ in the different stages of the experiment showed no significant effect of stage of the experiment. Apparently the greater difference between 3 clicks/sec and 18 clicks/sec, as compared to 3 and 9 clicks/sec, did not cause a greater difference between response rates in Stage 2 as compared to Stages 1 and 3. Response rates during $S-$ in the different stages of the experiment were also analyzed, and it was found that stage of the experiment did not significantly affect these rates either.

\section{DISCUSSION}

Higher click rates in this experiment were correlated with higher response rates and, in that sense, click frequency can be considered a stimulus intensity parameter. However, the results of this experiment raise several problems for the theories which seek to explain stimulus intensity effects. One such theory has been proposed by Hull (1952), and it suggests that response probability would be affected by the physical energy associated with the stimulus. Since 18 clicks/sec is more intense than 9 clicks/sec, response rate should have been higher during presentation of the former stimulus if the theory were correct. Since response rate was not higher during the $18 \mathrm{clicks} / \mathrm{sec}$ stimulus, other explanations must be considered. One alternative is the discriminability theory proposed by Perkins (1953) and Logan (1954). They argued that stimulus intensity should not be defined by the physical energy of the stimulus but as the difference between the conditioned stimulus and the background level of stimulation. Any application of this theory to our results would have to take account of difference in the discriminability of the $\mathrm{S}+\mathrm{s}$ from each other as well as from the $\mathrm{S}-$. If response rate were related to stimulus discriminability, the difference between response rate in the 3 clicks/sec $S+$ as opposed to the 9 clicks/sec $\mathrm{S}+$ would be smaller than the difference in response rate during the 3 clicks $/ \mathrm{sec} S+$ as compared to the 18 clicks/sec $\mathrm{S}+$. Response rate was higher in the more intense stimulus in both cases, but the magnitude of the difference in click frequency between the high and low intensity stimuli did not affect the magnitude of the difference in response rate between the two stimuli. The results of the Mostofsky et al study (1964) show that rats can easily discriminate the difference between 9 and $18 \mathrm{clicks} / \mathrm{sec}$. Therefore, the failure to find an effect when the difference between stimuli was increased cannot be explained as having been caused by too small an increase in the high intensity stimulus.

Finally, Grice (1968) has attempted to explain some stimulus intensity effects with a form of adaptation level theory. This theory perhaps could explain the fact that the difference in response rate during the $S+s$ did not increase when the difference in stimulus intensity was increased. It could be argued that the high and low intensity stimuli are always the same relative distance from the adaptation level, regardless of the magnitude of difference between the two stimuli, and that response rate simply reflected the stimulus position relative to the adaptation level. Thus, the difference between response rates in stimuli of two different intensities should not change when the magnitude of the intensity difference changes. However, the adaptation level of the $\mathrm{S}+\mathrm{s}$ should change and, when the adaptation level is farther from the $S-$, there should be either an increase in response rate during each $\mathrm{S}+$ or a decrease in response rate during $\mathrm{S}-$. Neither effect was observed in this experiment.

\section{REFERENCES}

Gray, J. A. Stimulus intensity dynamism. Psychological Bulletin, 1965, 63, 180-196.

Grice, G. R. Stimulus intensity and response evocation. Psychological Review, 1968, 75, 359-373.

Hull, C. L. A behavior system: An introduction to behavior theory concerning the individual organism. New Haven: $\mathrm{Y}$ ale University Press, 1952.

Logan, F. A. A note on stimulus intensity dynamism (V). Psychological Review, 1954, 61, 77-80.

Mostofsky, D. I., Shurtleff, D. A., \& Margolius, G. Comparative sensitivity of rats and humans to changes in auditory click rate. Journal of Comparative \& Physiological Psychology, 1964, 58, 436-440.

Perkins, C. D., Jr. The relation between conditioned stimulus intensity and response strength. Journal of Experimental Psychology, 1953, 46, 225-231.

Saunders, J. C. Behavioral discrimination of click intensity in the cat. Journal of Experimental \& Analy tical Behavior, 1969, 12, 951-957.

(Received for publication November 7, 1972.) 